\title{
Updating the path integrator through a visual fix
}

\author{
ANDREA S. GRIFFIN and ARIANE S. ETIENNE \\ University of Geneva, Geneva, Switzerland
}

\begin{abstract}
Mammals can navigate through path integration (dead reckoning) by updating their position on the basis of internal signals generated during locomotion, without using any external references. However, being open to cumulative errors, path integration remains functional over short excursions only, unless corrected by familiar landmarks. That such a corrective process may occur was examined in golden hamsters during hoarding excursions occurring in darkness, within a large open arena. The subjects proceeded from their peripheral nest to a feeding site on a platform. If the animals were rotated during food pouching, their subsequent homing behavior was disoriented, self-generated positional information having been disrupted. By contrast, when the subjects were rotated at the food source and then briefly presented with the familiar visual environment, they returned homeward, albeit not very precisely. Thus, the animals may have taken an approximate positional fix, or reset their internal compass only.
\end{abstract}

In recent years there has been an increasing interest in path integration. Also termed "dead reckoning," this navigation process allows the subject to update his/her position relative to a point of reference through a running computation of signals generated during locomotion. The route-based process requires the estimation of both distance and direction of travel and is commonly formalized as the computation of a vector that connects the subject to his/her point of departure. Thus, at the end of a sinuous outward journey, the subject tends to head back to the starting point of his/her excursion along a fairly direct path, as if following a homebound vector. In mammals, the computation of self-position can depend entirely on internally generated signals. Input from the vestibular system together with proprioceptive feedback and possibly efferent copies (copies of locomotor commands) continuously inform the subject of his/her locomotory movements and allow him/her to measure the angular and linear components of his/her pathway (Etienne, Maurer, \& Séguinot, 1996).

Path integration has the functional advantage of enabling an animal to navigate through a totally unfamiliar environment without losing track of its point of departure (e.g., its home). What's more, the system requires no learning and is probably functional as soon as a young individual begins making short exploratory excursions away from the nest (Etienne, Berlie, Georgakopoulos, \& Maurer, 1998).

A major drawback of path integration, however, is that the recurrent computation of position is subject to cumu-

\footnotetext{
This research was supported by Swiss NSF Grant 31-39311.93. Our warmest thanks are due R. Maurer for helpful discussions, R. Gallistel for commentaries on preliminary results, and $R$. Schumacher for his technical assistance. Correspondence should be addressed to A. Ettienne, Laboratoire d'Ethologie, Université de Genève, 54 route des Acacias, CH-1227 Carouge, Switzerland (e-mail: etienne@uni2a. unige.ch).
}

lative errors. The less precise the estimation of direction and distance of travel, the more route-based position information drifts. Measuring rotations and translations without external references, as it occurs in mammals, is affected by relatively large random errors and therefore leads to a rapid degradation of the position vector (for a review, see Maurer \& Séguinot, 1995). In particular, behavioral research in our laboratory has shown that in general golden hamsters (Mesocricetus auratus W.) are able to integrate only up to five active or two passive rotations, with particular individuals showing a higher degree of compensation than others (Etienne, Maurer, \& Saucy, 1988). Additionally, without external direction references, path-dependent information fades away quickly as the time interval between the onset and return of a journey increases. Finally, path integration models based on the observation of systematic homing errors suggest that this process depends on an approximate algorithm for updating position (see Etienne et al., 1996). It follows that path integration in general is affected by errors and that this is particularly the case if it is based on inaccurate first-order signals. In the latter case, path integration by itself remains reliable over short excursions only.

A completely different and therefore complementary navigational system consists of building stable associations between landmarks and places of functional importance. These associations and their mutual relationships may then be encoded on a maplike representation. Under these conditions, the subject can pilot through his/her environment by matching the currently perceived landmarks with their internal representation, and therefore locate himself/herself in relation to a goal on the map. Map navigation allows the planning of goal-directed trajectories in general and the choice of adequate paths in new situations (Gallistel, 1990; O'Keefe \& Nadel, 1978; Tolman, 1948). In contrast to path integration, however, this location-based navigation system is operant only in a familiar environment and when relevant landmarks are available. 
According to current theories of biological navigation systems, map navigation and path integration may cooperate in order to optimize navigation (Etienne, Maurer, et al., 1998; Gallistel, 1990). In particular, within a familiar environment, a subject may clear his/her path integrator from accumulated errors by taking a positional fix on the visual surroundings: By computing the relevant parameters from the currently perceived visual environment (see Discussion) and then matching the observed and mapped landmarks, the subject estimates his/her position on the map. Under these conditions, subjects may update the approximate route-based estimate of position with the exact location-based positional value. Episodic fixes (Gallistel, 1989, 1990) would therefore compensate for the inherent tendency of path integration to drift.

That familiar external references may update the path integrator has not yet been shown on the behavioral level. On the neurophysiological level, however, there is increasing evidence that in conditions in which the firing properties of hippocampal place cells and head direction cells in functionally related brain structures are subject to drift, cell activity may be updated by the presentation of the known visual environment (Goodridge \& Taube, 1995; see present Discussion).

The aim of the present research is to investigate whether golden hamsters (Mesocricetus auratus W.) update their path integrator when being briefly presented with a familiar visual environment. In our experimental conditions, each animal lives in the experimental arena throughout the testing period and can therefore establish stable associations between visual references and specific places and/or directions. In darkness, the subject returns from a foraging site to its peripheral nest by relying on internal path integration only - that is, without using any external reference. In light, however, homing is controlled by vision as well as by path integration. The hamsters' ability to navigate by means of landmarks and/or path integration creates an ideal situation for testing the cooperation between the two navigational systems (Etienne, Maurer, et al., 1998).

In the present experiments, each subject was led in darkness away from its nest on a hoarding excursion. At the feeding site, path integration was disrupted by submitting the animal to fast bidirectional rotations. Homing directions were analyzed when the animal had the opportunity to briefly sight the surrounding landscape at the end of the rotations (rotation + fix condition). Results were compared with a situation in which the subject was not given this opportunity (rotation + no fix condition). Under the hypothesis that the sighting of a familiar landmark panorama enables subjects to take a positional fix, we expected them to carry out home-directed returns in the rotation + fix condition and randomly orientated returns in the rotation + no fix condition.

\section{METHOD}

\section{Subjects}

Five laboratory-bred golden hamsters (Mesocricetus auratus W.), all adult females, participated in this experiment. All were bred in our laboratory. Four subjects had already been used in previous experiments and 1 was a naive subject. One animal was a pilot subject and underwent only two of three experimental conditions (no rotation + no fix and rotation + no fix; see General Procedure). Each individual lived alone in the experimental arena and nest box throughout the testing period. Animals were maintained under a 12:12-h light:dark cycle and tested at the beginning of the dark phase. They were fed ad lib with a variety of different food items.

\section{Apparatus}

The experimental arena (diameter, $220 \mathrm{~cm}$; height of peripheral wall, $45 \mathrm{~cm}$ ) was provided with a peripheral nest box where the subject established its granary. Between test sessions, the animal could move freely between the nest box and the arena through a small swinging door. A circular ceiling (diameter, $300 \mathrm{~cm}$ ) composed of white cloth was centered over the arena at $190 \mathrm{~cm}$ above the ground. Four light bulbs (intensity, $40 \mathrm{~W}$ ) positioned symmetrically above and around the cloth ceiling illuminated the arena and are referred to as the "room lights." Experimental trials took place in darkness and were recorded by an infrared (IR) sensitive video camera placed in the center of the ceiling and surrounded by a set of IR diodes (peak emission, $940 \mathrm{~nm}$; bandwidth, $45 \mathrm{~nm}$ ). The camera was connected to a video screen located in a room adjacent to the experimental hall. A small mobile circular platform (diameter, $26 \mathrm{~cm}$ ) could be lifted in and out and positioned anywhere within the arena. Its elevation above the ground was $3 \mathrm{~cm}$ and it could be rotated by means of a handle fixed to its center. The platform was removed from the arena between experimental sessions.

The experimental arena was situated in a large experimental hall $(8.5 \times 4.5 \times 3.2 \mathrm{~m})$ that provided the subject with an asymmetrical, heterogeneous visual background (four differently patterned walls, curtains, pillars, cleaning equipment, etc.). Along its long axis, the hall contained a second arena. The video camera and diodes could be displaced and positioned above the center of each arena on a rail system fixed to the ceiling of the hall.

\section{General Procedure}

The subject was introduced into the experimental arena on Day 1. It was shut into the nest box for $24 \mathrm{~h}$ to induce it to establish its nest and granary. Thereafter, it underwent one working session per day during the first quarter of the dark cycle. The subject's entire test period was divided into a training phase followed by an experimental phase. During the training phase, the subject was habituated to the experimental environment and procedures. The training phase contained from 5 to 10 sessions depending on the subject. In the experimental phase, the subject underwent $7-12$ experimental sessions. Thus each subject participated in the experiments for 15-30 days.

The general procedure of the experiments was to lead the animal from its nest to a food source where it was left to pouch food and then to return to its nest. This nest-centered back-and-forth excursion is called a "hoarding trip" and represents the fundamental unit of our experiments. The hoarding trip can be split into an outward and an inward, or return journey. To be selected for (and retained during) these experiments, the animals had to (1) establish their granary within the nest box and show intense motivation for hoarding (food pouching had to start as soon as the subject arrived at the food source) and (2) show nest-oriented homing in preliminary control trials (see below) and control trials during experiments. In total, for 5 subjects that underwent the experiments, 7 (i.e., about $60 \%$ ) did not fulfill one or both of these conditions and were eliminated. It should be noted that in all our experiments on homing, a high proportion of hamsters have to be discarded because the test conditions are extremely demanding.

Throughout the experiments, pink noise was diffused into the arena from four loudspeakers placed symmetrically around the arena to mask all possible acoustical cues. The positions of the loudspeakers were permuted from one session to another. Olfactory and tactile trails were eliminated by stirring a thick $(7-\mathrm{cm})$ layer of 
sawdust on the floor of the arena. The sawdust was always stirred before the beginning of a session. During the experimental phase, the sawdust was also stirred after preliminary control trials (see below) before any test trials were run. Bands made of pliable metal (length, $120 \mathrm{~cm}$; height, $20 \mathrm{~cm}$; width, $0.2 \mathrm{~cm}$ ) were placed along the inner walls of the arena so as to mask all olfactory markings. The bands were removed between sessions. Their positions were permutated from session to session. Geomagnetic cues did not seem to influence our animals' homing behavior and were not altered (Etienne, Maurer, Saucy, \& Teroni, 1986). That the animals used neither (1) intramaze nor (2) extramaze orientational cues was also verified post hoc by the fact that (1) they did not retrace their outward path on return to the nest and $(1,2)$ they were no longer oriented in the rotation + no fix condition (see below, on experimental excursions, and see Results).

\section{Training Phase}

During training sessions, the platform was baited with a pile of hazelnuts and placed before each hoarding trip anywhere within the arena but at least $30 \mathrm{~cm}$ away from the peripheral wall and $35 \mathrm{~cm}$ away from the center of the arena. The subject was induced to follow a lure illuminated by a small red light to the platform and to pouch food on the platform; thereafter it returned to its nest. As the platform was moved before each hoarding trip, the length and the direction of the outward journey varied at each hoarding excursion. The journeys took place under room lighting and without masking of background noise until the animal became more confident. Thereafter, the room lights were switched off and pink noise was diffused. This training phase ended when the animals were habituated to the platform and the pink noise. Subjects then underwent the experimental phase.

\section{Experimental Phase}

At the beginning of an experimental session, the subject was briefly refamiliarized with the procedure used during training sessions (see Training Phase). As soon as the animal began carrying out swift hoarding excursions with direct returns to the nest under maximal pink noise intensity, room lights were switched off and the experimental session started.

Preliminary and referential excursions. An experimental session started with up to eight preliminary control trials, referred to as preliminary excursions. These allowed us to make sure that the animal was sufficiently motivated and correctly oriented within the arena to run test trials. The platform was moved before each hoarding excursion within the same limits of the arena used in training sessions (see Training Phase). On preliminary trips, the subject had to carry out three nest-oriented returns within four successive excursions. A nest-oriented return was defined as follows: On returning from the food location, the subject entered the peripheral annular zone of the arena (see Encoding and Evaluation of Trajectories) within an area limited by a $90^{\circ}$ angle the summit of which coincided with the food location and whose bisecting line intersected with the nest. Experimental trials were started if the subject fulfilled the criteria within a maximum of eight preliminary control trials; if it did not, the experimental session was put off for $24 \mathrm{~h}$. The sawdust was always thoroughly stirred after the preliminary excursions, before any experimental trials were run.

Thereafter, in the course of the experimental session the subject had to carry out two nest-orientated returns within four excursions, referred to as referential excursions, between every experimental trial. Thus the orientation and the motivation of the subject were constantly monitored throughout the experimental session.

Experimental excursions. For the experiment seven hoarding locations were defined within the arena. They were all located at a radial distance of $50 \mathrm{~cm}$ from the center of the arena and $60 \mathrm{~cm}$ from the nearest section of the peripheral wall and were spaced at $45^{\circ}$ intervals (see Figure 2). The remaining eighth location on the circle was considered to be too close to the nest exit and was therefore not used.

The experiment contained three conditions: In the no rotation + no fix and the rotation + no fix conditions, the subject carried out the whole hoarding excursion in darkness. In the rotation + fix condition, the room lights were briefly switched on after the subject had been rotated. Total hoarding duration on the platform was $25-32 \mathrm{sec}$ in all conditions.

In the no rotation + no fix condition, the platform was placed on one of the seven predefined locations. A pile of hazelnuts was placed on the platform. The subject was led in obscurity from its nest onto the platform and pouched the hazelnuts. The outward trajectory was always the shortest path from the nest to the hoarding location (Figure 2). After 25-32 sec, the subject had collected all the available food items and returned to its nest.

In the rotation + no fix condition, the subject was again led in darkness from its nest to the same location, but while pouching food it was submitted to five full rotations in one direction, five full rotations in the other, and one-quarter rotation in the first direction. The first direction of rotation was alternated from trial to trial. The average speed of rotation was 0.8 rotations per second. Total rotation time was approximately $12 \mathrm{sec}$. Care was always taken that the animal hoarded continuously during rotations and for a further $15-20 \mathrm{sec}$ after the rotations.

In the rotation + fix condition, the subject was again led in darkness to the same hoarding location and rotated during food uptake, but this time the room lights were switched on for 10 sec immediately after the rotations. Darkness was then reestablished. After hoarding for approximately 5-12 sec longer, the animal returned to its nest.

Each of the seven locations was used twice in each of the three conditions. Thus, subjects underwent a total of 42 experimental trials distributed over 7 to 12 experimental sessions, depending on whether they fulfilled the test conditions or not (see Experimental Phase). The order in which the locations were used was randomized, but animals were always tested in the three conditions at a given location in the order no rotation + no fix, rotation + no fix, and rotation + fix. Between two successive test trials occurring in different conditions, the hoarding location was altered. Two experimental trials at the same location were always separated by up to four referential excursions (see Experimental Phase) and an experimental trial at one of the other seven locations.

\section{Encoding and Evaluation of Trajectories}

All return trajectories were traced from the video screen (see Apparatus) onto transparent paper. Return itineraries were encoded by means of a mobile grid centered at the hoarding location and made of four concentric circles and $3610^{\circ}$ sectors (Figure 1). The radius of the inner circle was $20 \mathrm{~cm}$; the outer annuli increased by $15-\mathrm{cm}$ intervals. The center line of Sector 1 intersected with the nest and was assigned the direction $0^{\circ}$. This was called the reference direction. Sectors were numbered clockwise from 1 to 36 . To encode a return path, we noted the sector in which the animal had crossed each of the successive concentric circles; each return path was therefore transcribed into a sequence of 2-4 numbers. A $15-\mathrm{cm}$-wide peripheral zone of the arena was defined as the peripheral annular zone of the arena. The intersection of a trajectory with a concentric circle on the mobile grid was counted only if the intersection was not located within the peripheral annular zone of the arena. If the intersection lay within this zone, only the intersection with the previous concentric circle was counted. This precaution was taken because the hamsters might otherwise have picked up olfactory information on the arena wall during their final approach to the periphery. Trajectories were therefore not analyzed within the peripheral area even if the arena walls were covered with bands (see General Procedure). 


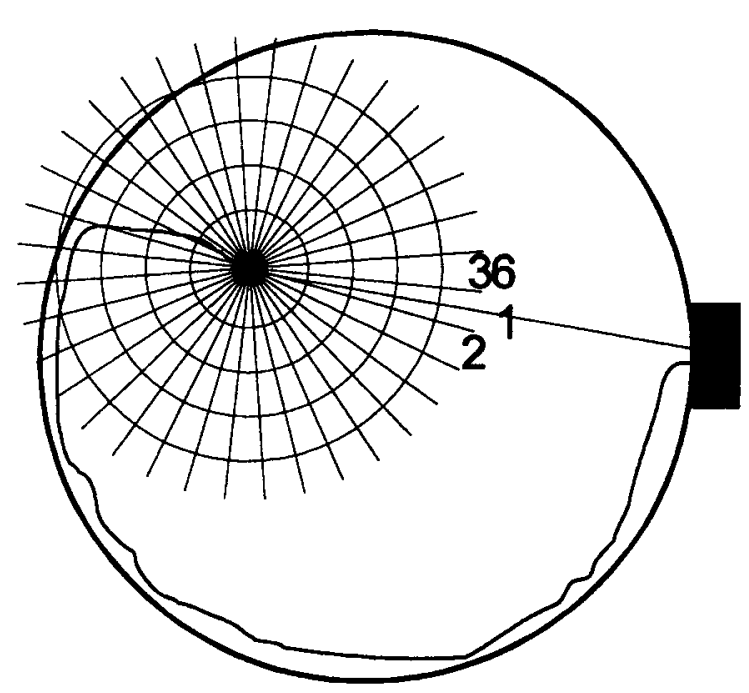

Figure 1. Encoding of return itineraries traced from the video screen. The large circle represents the arena. The nest is represented by a filled rectangle to the right of the arena. The mobile grid used for encoding the return paths was centered at the hoarding location and the $0^{\circ}$ reference direction (middle of Sector 1 ) was lined up with the nest direction. Depicted here is an example of a return path leading toward the peripheral wall of the arena and thus intersecting with three concentric circles (for further details, see text).

The homing time was measured in terms of the time interval between leaving the hoarding location and reaching the nest entrance.

\section{Statistical Analysis}

Since the seven hoarding locations used in this experiment were located at $60 \mathrm{~cm}$ from the nearest section of the peripheral wall and since return trajectories were not analyzed within the peripheral zone of the arena (see above), itineraries intersected with two $(35 \mathrm{~cm}$ from the hoarding location) to four ( $65 \mathrm{~cm}$ from the hoarding location) concentric circles, depending on whether the itinerary led toward or away from the peripheral wall of the arena. The analysis of the subjects' return direction beyond the second concentric circle led to the loss of the trajectories in which the subject headed for the periphery (Figure 1). Therefore, for all experimental conditions, the subjects' orientation was analyzed with respect to the transgression of the second circle, at a distance of $35 \mathrm{~cm}$ from the hoarding location. Under the hypothesis that the returns were nest oriented in the no rotation + no fix and the rotation + fix conditions, the trajectories observed in these conditions were not analyzed further away from the hoarding location. In the rotation + no fix condition, however, the data were also analyzed, whenever possible, with respect to the fourth concentric circle (65 $\mathrm{cm}$ from the hoarding location). This analysis enabled us to verify that rotating the subjects caused a complete disruption of internal cues and not a delayed adjustment to a nest-directed final orientation.

For each subject, the returns from the seven hoarding locations in a given experimental situation were pooled. Circular statistics (Batschelet, 1981) were used to evaluate the homing directions during the experimental trials. The Rayleigh first-order test was used to determine whether the subjects were significantly oriented in each of the three experimental conditions. Stephens's confidence interval was determined for each subject and condition and used to test whether the subject's mean orientation differed significantly from the $0^{\circ}$ reference direction (nest direction). Mean group orientation in each condition was tested for significance by using Moore's second-order test. Student parametric $t$ test was applied to compare group average homing times between conditions.

\section{RESULTS}

In the experimental conditions in which the animals were neither rotated nor presented with the landscape (no rotation + no fix), the subjects were all significantly oriented toward the nest at $35 \mathrm{~cm}$ from the hoarding location (Table 1 and Figure 2). No subject showed a mean orientation that deviated more than $34^{\circ}$ from the $0^{\circ}$ reference direction (i.e., the nest). In no case was the deviation statistically significant (Table 1). Mean orientation over subjects was $-12^{\circ}$ and highly significant (Moore's test, $D^{*}=1.29, p<.01$; Figure 2).

In contrast, when submitted to fast bidirectional rotations while collecting food on the platform (rotation + no fix), all subjects were randomly oriented at $35 \mathrm{~cm}$ from the hoarding location (Table 1 and Figure 2). Mean orientation over subjects was not significant (Moore's test, $D^{*}=0.25, p>.05$; Figure 2). Four of 5 subjects were still not oriented at $65 \mathrm{~cm}$ from the hoarding location (Table 2). This result confirmed that rotating the subjects completely disrupted internally generated positional information and did not simply delay the animals' adjustment to a nest-directed final orientation. Furthermore, 4 of 5 subjects took longer to reach home when they had been submitted to rotations than when not (Table 1, compare no rotation + no fix with rotation + no fix). Group average homing time was significantly longer in the situation with than in the situation without rotations (Student $t$ test, $t=3.12, p<.05$ ).

When the subjects were submitted to rotations and then presented with the visual panorama during $10 \mathrm{sec}$ (rotation $+\mathrm{fix}$ ), the mean homing direction was $-19^{\circ}$ and was significant (Moore's test, $D^{*}=1.14, p<.05$; Figure 2). With regard to first-order results, 3 of the 4 animals achieved oriented return itineraries. Two animals homed significantly toward the nest, and 1 subject deviated significantly counterclockwise by $40^{\circ}$ from the nest (Female 3, Stephens's confidence interval $=33^{\circ}$ ). The nonsignificant returns of the 4th subject (Female 2) can be explained by the small number of trials (Table 1 and Figure 2). All subjects took less time to reach the nest entrance when they had viewed the panorama after the rotations than when not (Table 1). Group average homing time was significantly shorter in the rotation + fix than in the rotation + no fix situation (Student $t$ test, $t=7.82, p<.01$ ).

Taken together, these results show that the subjects used the available visual information to select a preferential return direction. However, the scatter of the homing directions chosen by particular individuals remained higher after the fix than in the no rotation + no fix condition. Further, it seems that the selected homing direction did not always correspond exactly to that of the nest direction. This appeared more clearly in a detailed analysis of the return itineraries from particular subjects. 
Table 1

The Orientation of Subjects in the Three Experimental Conditions at a Distance of $35 \mathrm{~cm}$ From the Hoarding Site

\begin{tabular}{|c|c|c|c|c|c|c|c|}
\hline \multirow[b]{2}{*}{ Condition } & \multirow[b]{2}{*}{ Subject } & \multirow[b]{2}{*}{$N$} & \multicolumn{2}{|c|}{ Mean Vector } & \multirow[b]{2}{*}{$\pm \delta\left({ }^{\circ}\right)$} & \multirow[b]{2}{*}{$\mathrm{T}(\mathrm{sec})$} & \multirow[b]{2}{*}{$\operatorname{Tm}(\mathrm{sec})$} \\
\hline & & & Direction $\left(\theta^{\circ}\right)$ & Length $(r)$ & & & \\
\hline \multirow[t]{5}{*}{ No rotation + no fix } & 1 & 14 & -21 & $0.64 \uparrow$ & 32 & 10.1 & \\
\hline & 2 & 8 & -34 & $0.67^{*}$ & 43 & 13.3 & \\
\hline & 3 & 13 & 10 & $0.88 \dagger$ & 18 & 7.1 & 8.5 \\
\hline & 4 & 15 & 0 & $0.86 \dagger$ & 16 & 6.1 & \\
\hline & 5 & 14 & -16 & $0.87 \dagger$ & 17 & 5.9 & \\
\hline \multirow[t]{5}{*}{ Rotation + no fix } & 1 & 14 & 49 & 0.20 & $\infty$ & 14.4 & \\
\hline & 2 & 8 & -161 & 0.26 & $\infty$ & 12.7 & \\
\hline & 3 & 14 & -9 & 0.13 & $\infty$ & 12.4 & 13.1 \\
\hline & 4 & 15 & -89 & 0.40 & $\infty$ & 12.4 & \\
\hline & 5 & 14 & 47 & 0.21 & $\infty$ & 13.7 & \\
\hline \multirow[t]{4}{*}{ Rotation + fix } & 2 & 7 & -44 & 0.63 & - & 9.2 & \\
\hline & 3 & 14 & -40 & $0.63 \dagger$ & 33 & 8.1 & 7.7 \\
\hline & 4 & 15 & 5 & $0.75 \dagger$ & 23 & 6.8 & \\
\hline & 5 & 14 & 3 & $0.53^{*}$ & 43 & 6.7 & \\
\hline
\end{tabular}

Note-Return trajectories from all seven hoarding locations were pooled for statistical analysis. $N$, number of trials. The $0^{\circ}$ reference direction points toward the nest. Positive angles were measured clockwise. $\delta$, Stephens's confidence interval for the mean angle (confidence coefficient $Q=0.95$ ), $\infty$, an infinitely large interval. Sample size for Female 2, which ceased to fulfill the test conditions (see Experimental Phase) during the experiment and had to be discarded, was too small in the rotation + fix condition to allow determination of $\delta$. T, homing time (time between leaving the hoarding location and touching the nest entrance). Tm, mean homing time for the experimental group. Female 1 was a pilot subject and was not tested in the rotation + fix condition. ${ }^{*} p<.05 . \quad{ }^{\dagger} p<.01$ (Rayleigh test).

Figure 3 shows that in the no rotation + no fix condition, the return directions of all subjects pointed in general toward the nest. Furthermore, all subjects entered the peripheral zone of the arena on both sides of the nest entrance, regardless of the location of the food source. In contrast, in the rotation + fix condition, the homing directions tended to scatter more widely around each side of the nest, and the subjects tended strongly to enter the peripheral zone of the arena on the same side as that from which they had departed. An exception was Female 5 in trials in which the food source was located counterclockwise from the nest.

In summary, the presentation of the landscape after having disrupted path integration through rotations

\section{Outward journey}

\section{Return journey}

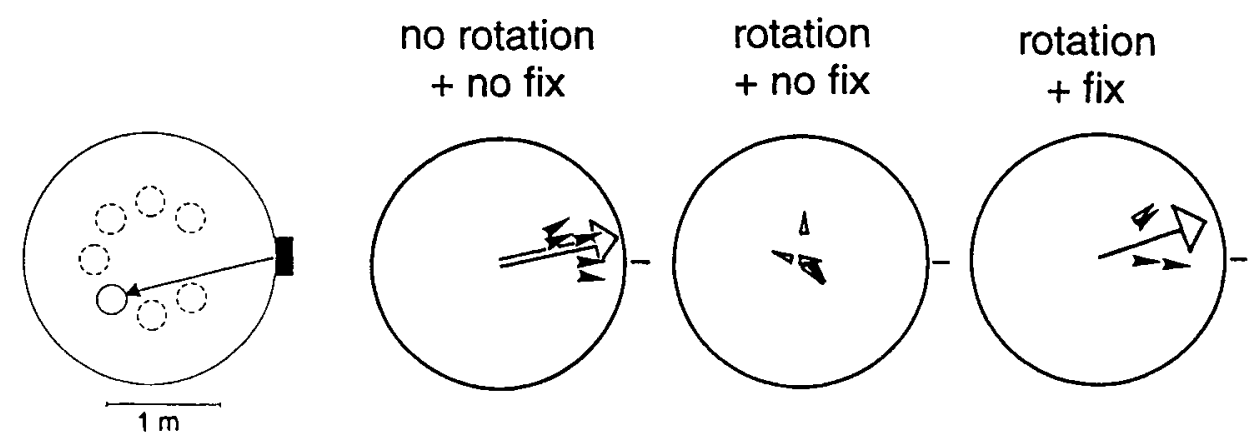

Figure 2. Testing the effect of a visual fix. Left: Each subject was tested twice at each of seven hoarding locations within the arena. The outward trajectory was always the shortest path between the nest (filled rectangle) and the platform (full circle). The platform was moved between hoarding locations (dashed circles). Right: first- and second-order vectors representing the subjects' return direction at a distance of $35 \mathrm{~cm}$ from the hoarding location. Return itineraries from the seven hoarding locations were pooled. The return vectors are drawn with respect to the nest as $0^{\circ}$ reference direction. The small arrowheads indicate the mean homing direction for each subject (filled arrowheads, $p<.05$ or $<.01$; empty arrowheads, $p>.05$; Rayleigh test). The large open arrows indicate a significant (single line, $p<$ .05 ; Moore's test) or a highly significant (double line, $p<.01$; Moore's test) mean homing direction for the whole experimental group. 
Table 2

Orientation of Subjects in the Rotation + No Fix Condition at a Distance of $65 \mathrm{~cm}$ From the Hoarding Site

\begin{tabular}{ccccc}
\hline & & \multicolumn{2}{c}{ Mean Vector } & \\
\cline { 3 - 4 } Subject & $N($ Ntot $)$ & Direction $\left(\theta^{\circ}\right)$ & Length $(r)$ & $\pm \delta\left({ }^{\circ}\right)$ \\
\hline 1 & $12(14)$ & -7 & 0.33 & $\infty$ \\
2 & $7(8)$ & -178 & 0.24 & $\infty$ \\
3 & $8(14)$ & -45 & 0.28 & $\infty$ \\
4 & $9(15)$ & -41 & $0.63^{*}$ & $\infty$ \\
5 & $8(14)$ & 78 & 0.30 & $\infty$ \\
\hline
\end{tabular}

Note-(Ntot), total number of trials. $N$, number of trials in which trajectories aimed away from the periphery of the arena and could therefore be analyzed at $65 \mathrm{~cm}$ from the hoarding location (see Method, Statistical Analysis). For further explanation, see note to Table $1 .{ }^{*} p<.05$.

clearly reestablished homing behavior. However, in general the return directions remained less accurate in these conditions than when subjects homed through path integration only. Possibly the subjects tended to compute the general direction of the nest rather than an accurate vector from self-position to nest.

\section{DISCUSSION}

Path integration based on internal signals remains functional over small travel distances only. This means that during the exploration of a new environment, the animal has to return frequently to a fixed point of reference to reset its path integrator. In a familiar environment, path integration may go on over longer distances, provided that it is corrected by familiar references. Among these references, visual landmarks must play a privileged role because they yield precise positional information that can be perceived on the spot as well as from a distance.

Our results clearly show that the subjects were able to gain homeward information during a brief sighting of their familiar visual environment after having lost track of their position relative to the nest. This was shown by the mean homing directions from particular individuals and from the whole experimental group (Table 1 and Figure 2), by the general shape of the homing trips (Figure 3), and by the shorter homing times in the rotation + fix condition compared with those in the rotation + no fix condition (Table 1). The improvement of the homing behavior through the fix implies that the subjects were able to retain and use the intermittently presented visual information while they continued to collect food in darkness before initiating their return to the nest. However, performance in terms of homing directions and scatter remained inferior to that observed in trials occurring in continuous darkness, where path integration had not been disrupted (Table 1 and Figure 2). The brief presentation of the landmark panorama seemed to allow the subjects to compute a general homing direction rather than a precise relationship between their position at the feeding site and their nest (Figure 3).

That the subjects may have taken up directional rather than positional information when sighting the visual en- vironment was suggested by a further experiment in which subjects were led on a two-legged outward journey from the nest to a feeding place (unpublished results). At the end of the first outward leg, the subject was rotated and then given the opportunity to make a visual fix. To choose the correct homing direction at the end of the outward trip, the subject had to reestablish its position in relation to the nest during the fix and then continue to update its position during the second outward leg. Subjects' homing patterns were not clear-cut. However, in certain categories of trials, particular subjects showed a lower scatter of the homing directions and returned more in the general direction of the nest when they had sighted the visual panorama than when they had not. The partial improvement of the animals' return could be explained by the gain of directional instead of positional information during the visual fix.

The present results therefore open up the following alternatives. (1) Hamsters are capable of locating themselves in a known environment through intermittent fixes and of feeding this information into their path integrator in order to resume dead reckoning. Our experimental conditions, however, did not require precise homing behavior and/or did not allow the subjects to establish their position with respect to the nest during the presentation of the visual surrounding. (2) Our subjects are capable of getting and/or using general compass information only by sighting the familiar environment. Thus, they update their orientation but not their position with respect to a directional reference during further (active) rotations at the feeding place and possibly also during further translations, as suggested by the above-mentioned experiment.

In regard to the first alternative, a navigator may establish his/her position (for general considerations, see Gallistel, 1990) by sighting from his/her current position a single familiar landmark and by computing its allocentric bearing and distance. At the other extreme, the navigator can locate himself/herself in a known environment with the help of a set of point landmarks and their mutual spatial relationships and/or with respect to surfaces that determine the general shape of the known environment (O'Keefe \& Burgess, 1996). Local views (i.e., the perception of the environment from a current viewpoint) may describe unique positions (Leonard \& McNaughton, 1990; Touretzky \& Redish, 1996) even if the relationships between particular cues are apprehended in a more general topological manner than according to metric relationships. To head from his/her current position to a goal, the subject has further to relate his/her position and heading to the goal location. This can be done through a general matching process between the perceived visual environment and its stored representation, or by the pairwise combination of perceived and memorized vectors. Gerbils, for instance, seem to plan a goal-directed trajectory by subtracting the memorized goal-to-landmark vector from the currently perceived vector, which relates their own position to the landmark (Collett, Cartwright, \& Smith, 1986). 


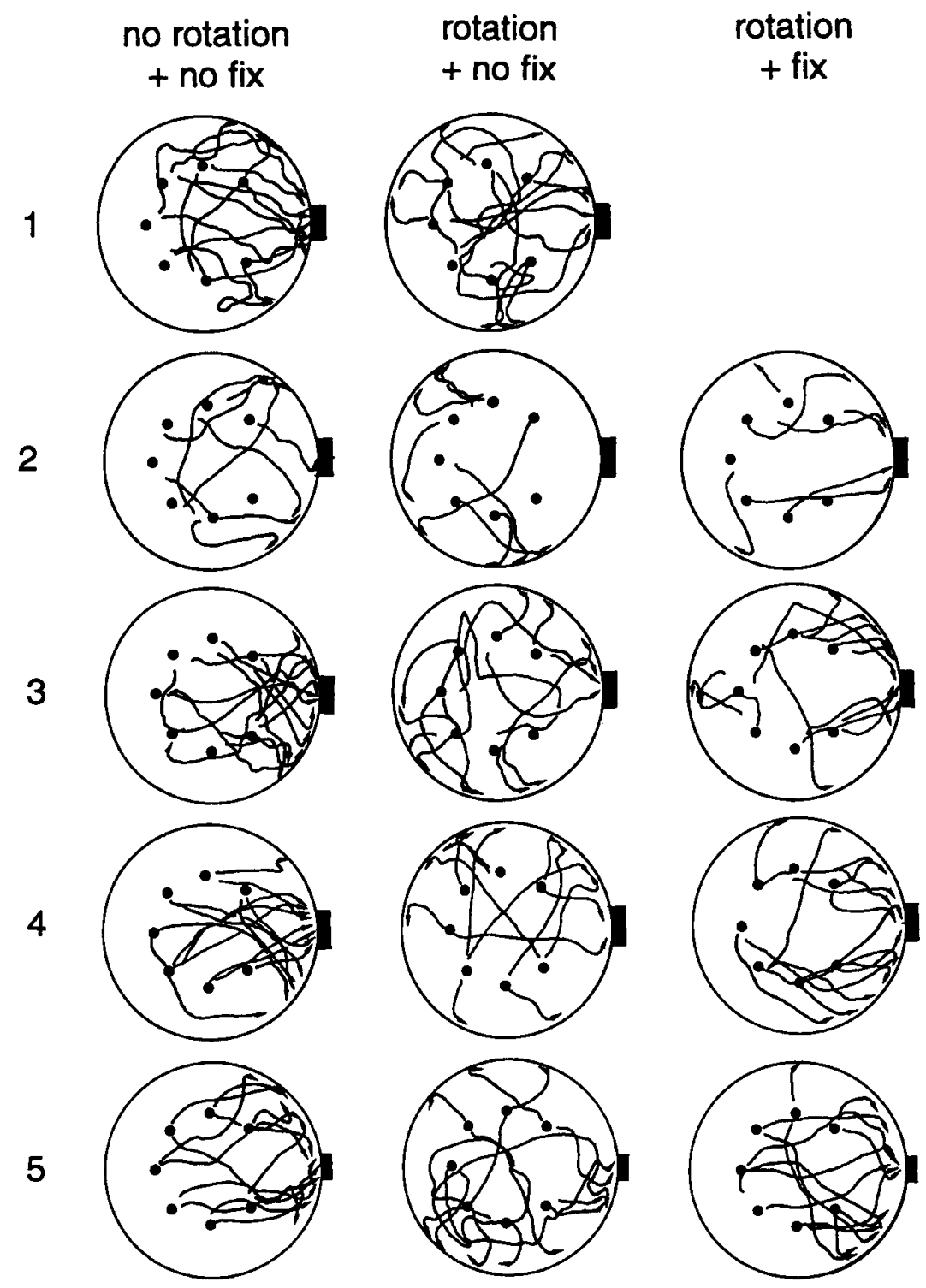

Figure 3. Detailed return trajectories of 5 (no rotation + no fix and rotation + no fix) and 4 subjects (rotation + fix). Female 1 was a pilot subject and underwent only two of three experimental conditions (no rotation + no fix and rotation + no fix, see General Procedure). Female 2 ceased to fulfill the test conditions (see Experimental Phase) during the experiment and had to be discarded. For further information, see Table 1 and Figure 2.

Planning the trajectory to the goal by a general matching process as well as by vector addition requires the animal to relate the memorized and currently perceived landmark bearings to the same reference direction. Rodents may derive this compass reference from the visual environment by using, for instance, single salient cues located at a distance (O'Keefe \& Nadel, 1978; Touretzky \& Redish, 1996), the geometric slope of the environment that the animal derives from the general cue distribution (O'Keefe, 1991), the general shape of an asymmetric environment, or the geometrical arrangement of a set of landmarks (Collett et al., 1986). Inasmuch as the matching process also requires distance information, candidates for visual distance perception in rodents are the apparent size of known landmarks as well as dynamic depth cues such as motion parallax and looming (see Etienne, Joris, Maurer, \& Teroni, 1990).

To summarize, rodents seem to have the necessary perceptual and computational abilities for taking a position fix in adequate visual conditions according to the current literature. Further, our earlier data show that locationbased information and route-based spatial information interact in hamsters. Thus, visual references may reset a drifting path integrator. Given these positive assump- 
tions, the following considerations may explain the approximate homing directions that we observed in the rotation + fix conditions.

First, our experimental conditions may not have been sufficiently compelling to motivate the subjects to plan an accurate homing trajectory. An approximate homeward direction followed by a short walk along the peripheral arena wall allowed the subjects to return safely and fairly rapidly to their peripheral nest. Second, the visual information the animals could use may not have been adequate enough to allow them to compute their position. The relatively high arena walls and the artificial ceiling above the arena restricted the panoramic view of the surroundings.

According to our second general hypothesis, hamsters are not equipped to make a positional fix and/or to reset their path integrator through visual information. Instead, they derive a general reference direction from the sighting of extramaze cues and update the representation of their head and body orientation with respect to this reference during further rotations around the food source, in darkness.

The capacity for updating direction independently from position was already suggested by our initial homing experiments in hamsters (Etienne, 1980). In certain conditions, our subjects homed in a constant compass direction, as if their homebound vector depended on the integration of rotations, but not of translations, during the outward journey. That the animals were tested subsequently without the availability of any external reference and still chose a constant homing direction (Etienne, Maurer, Saucy, \& Teroni, 1986) suggests the existence of an "internal compass."

The existence of such a compass has been confirmed recently by neurophysiological data. Rats possess not only hippocampal place cells that fire when the animal is located at a given place of a familiar environment (for reviews, see McNaughton et al., 1996; Muller, Kubie, Bostock, Taube, \& Quirk, 1991), but also head direction cells (for reviews, see Muller, Ranck, \& Taube, 1996; Taube, Goodridge, Golob, Dudchenko, \& Stackman, 1996). These units from the posterior subiculum and other functionally related brain areas function as an integrated directional system, each of the cells showing a peak activity when the animal is heading in a given direction, independently of its location.

As in the case of place cells (Markus, Barnes, McNaughton, Gladden, \& Skaggs, 1994; McNaughton et al., 1996; McNaughton, Leonard, \& Chen, 1989; Quirk, Muller, \& Kubie, 1990), the activity of head direction cells is controlled to a large extent by visual references, but goes on for a certain time when visual references are no longer available. Convergent data show that place cells (see McNaughton et al., 1996) and head direction cells (see Muller et al., 1991) are in fact also driven by inertial stimuli and somatosensory feedback (i.e., by a set of signals generated through the animal's own rota- tions and translations [place cells] or rotations only [head direction cells]).

More to the point, Goodridge and Taube (1995) showed that drifting postsubicular and thalamic head direction cells can be reset by familiar visual stimuli. During a first recording session, rats walked freely around a circular environment with a white cue card taped to the inside wall as their only visual reference. The head direction cells established a preferred firing direction in relation to the cue card. In a second session, the cue card was removed. If a particular cell's preferred direction drifted more than $30^{\circ}$, the card was reintroduced in its original location. In this case, the cell's preferred direction generally shifted back to its initial orientation as determined by the cue card. If a particular cell's preferred direction remained stable during the session with no cue card, the card was reintroduced in a rotated position. Now, the cell's preferred direction rotated with the card. Further data confirm that visual information can set and reset the preferred direction of head direction cells.

Our present research aims at answering the questions raised by our initial results. We are trying to establish whether our animals can make positional or directional fixes by testing them in more stringent spatial conditions and in different visual environments. Furthermore, our experiments imply outward paths of different shapes and less radical procedures for degrading self-generated spatial information before the presentation of visual references. In natural conditions, location-based information and route-based information must complement each other in a more continuous manner, with visual fixes intervening before internal position and direction systems are out of function.

\section{REFERENCES}

Batschelet, E. (1981). Circular statistics in biology. London: Academic Press.

Collett, T. S., Cartwright, B. A., \& SMrTh, B. A. (1986). Landmark learning and visuo-spatial memories in gerbils. Journal of Comparative Physiology $A, 158,835-851$.

ETIENNE, A.S. (1980). The orientation of the golden hamster to its nestsite after the elimination of various sensory cues. Experientia, 36, 1048-1050.

etienne, A. S., Berlie, J., Georgakopoulos, J., \& Maurer, R. (1998). The role of dead reckoning in navigation. In S. Healy (Ed.), Spatial representation in animals (pp. 54-68). Oxford: Oxford University Press.

Etienne, A. S., Joris, S., Maurer, R., \& Teroni, E. (1990). Enhancing the impact of visual extra-maze cues in a spatial orientation task. Behavioural Brain Research, 38, 199-210.

Etienne, A. S., Maurer, R., Berlie, J., Derivaz, V., GeorgakopouLOS, J., GRIFFIN, A., \& Rowe, T. (1998). Cooperation between dead reckoning (path integration) and external position cues. Journal of Navigation, 51, 23-34.

Etienne, A. S., Maurer, R., \& Saucy, F. (1988). Limitations in the assessment of path dependent information. Behavior, 106, 81-111.

Etienne, A. S., Maurer, R., Saucy, F., \& Teroni, E. (1986). Shortdistance homing in the golden hamster after a passive outward journey. Animal Behaviour, 34, 696-715.

Etienne, A. S., Maurer, R., \& SÉGuinot, V. (1996). Path integration 
in mammals and its interaction with visual landmarks. Journal of $E x$ perimental Biology, 199, 201-209.

Gallistel, C. R. (1989). Animal cognition: The representation of space, time and number. Annual Review of Psychology, 40, 155-189.

GALLISTEL, C. R. (1990). The organization of learning. Cambridge, MA: MIT Press.

GoOdRidge, J. P., \& TAUbe, J. S. (1995). Preferential use of the landmark navigational system by head direction cells in rats. Behavioral Neuroscience, 109, 49-61.

LEONARD, B., \& McNaughton, B. L. (1990). Spatial representation in the rat: Conceptual, behavioral, and neurophysiological perspectives. In R. P. Kesner \& D. S. Olton (Eds.), Neurobiology of comparative cognition (pp. 363-422). Hillsdale, NJ: Erlbaum.

Markus, E. J., Barnes, C. A., McNaughton, B. L., Gladden, V. L., \& SKAGGS, W. E. (1994). Spatial information content and reliability of hippocampal CA1 neurons: Effects of visual input. Hippocampus, 4, 410-421.

MAURER, R., \& SÉGUiNot, V. (1995). What is modelling for? A critical review of the models of path integration. Journal of Theoretical Biology, 175, 457-475.

McNaughton, B. L., Barnes, C. A., Gerrard, J. L., Gothard, K., Jung, M. W., KNIERIM, J. J., Kudrimoti, H., QIN, Y., Skaggs, W. E., SuSTER, M., \& Weaver, K. L. (1996). Deciphering the hippocampal polyglot: The hippocampus as a path integration system. Journal of Experimental Biology, 199, 173-185.

McNaughton, B. L., Leonard, B., \& Chen, L. (1989). Corticalhippocampal interactions and cognitive mapping: A hypothesis based on reintegration of the parietal and inferotemporal pathways for visual processing. Psychobiology, 17, 230-235.
Muller, R. U., Kubie, J. L., Bostock, E. M., TAube, J. S., \& QuirK, G. J. (1991). Spatial firing correlates of neurons in the hippocampal formation of freely moving rats. In J. Paillard (Ed.), Brain and space (pp. 296-333). Oxford: Oxford University Press.

Muller, R. U., Ranck, J. B., \& TAUBE, J. S. (1996). Head direction cells: Properties and functional significance. Current Opinion in Neurobiology, 6, 196-206.

O'KeEFE, J. (1991). The hippocampal cognitive map and navigational strategies. In J. Paillard (Ed.), Brain and space (pp. 273-295). Oxford: Oxford University Press.

O'KEEFE, J., \& BURGESS, N. (1996). Geometric determinants of the place fields of hippocampal neurons. Nature, 381, 425-428.

O'KeEFE, J., \& NADEL, L. (1978). The hippocampus as a cognitive map. Oxford: Oxford University Press, Clarendon Press.

QUiRK, G. J., Muller, R. U., \& KUBIE, J. L. (1990). The firing of hippocampal place cells in the dark reflects the rat's recent experience. Journal of Neuroscience, 10, 2008-2017.

TAube, J. S., Goodridge, J. P., Golob, E. J., Dudchenko, P. A., \& Stackman, R. W. (1996). Processing the head direction cell signal: A review and commentary. Brain Research Bulletin, 40, 477-486.

Tolman, E. C. (1948). Cognitive maps in rats and men. Psychological Review, 55, 189-208.

TouretZKY, D. S., \& Redish, A. D. (1996). A theory of rodent navigation based on interacting representations of space. Hippocampus, $\mathbf{6}$, 247-270.

(Manuscript received November 19, 1997; revision accepted for publication March 18, 1998.) 